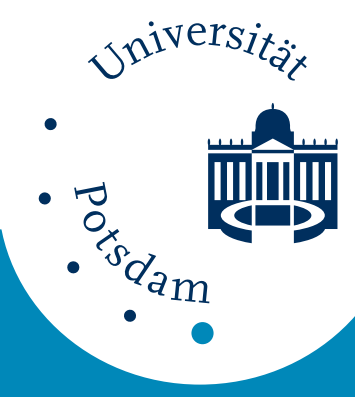

Universität Potsdam

Olaf Schmidtmann

Modelling of the interaction of lower and higher modes in two-dimensional MHD-equations

NLD Preprints ; 17 


\title{
Modelling of the interaction of lower and higher modes in two-dimensional MHD-equations
}

\author{
()laf Schmidtmann \\ Max-Planck-Gruppe Nichtlineare Dynamik, Universität Potsdam, \\ PF 601553, D-14415 Potsdam, Germany
}

Key words : MHD-equations, approximate inertial manifolds

\section{Introduction}

The present paper is related to the problem of approximating the exact solution to the magnetohydrodynamic equations (MHD). The behaviour of a viscous, incompressible and resistive fluid is exemined for a long period of time.

We follow the strategy introduced by several authors to approximate the solutions of the Navier-Stokes-Equations (NSE). The existence of a finite dimensional global attractor and a finite number of determining modes of the two-dimensional NSE ( $\mathrm{cf}$.[4],[5] ) shows that the long time behaviour of these equations is determined by a finite number of parameters.

This justifies the approximation of the solutions by the linear Galerkin approximation associated to the eigenvectors of the Stokes operator.(cf.[4]) In order to obtain a better approximation it was pointed out that the long time behaviour of two-dimensional turbulent flows is mainly controlled by a finite number of modes related to the Stokes operator and that the higher modes remain small for a large time. ( cf.[6] )

Let $u$ be the solution to the abstract evolution equation in an appropriate Hilbertspace

$$
\frac{d u}{d t}+A u+B(u)=f
$$

where $A$ is the Stokes operator and $B$ is a nonlinear quadratic operator. (0.1) can be written as a coupled system of equations for $p$ and $q$, where $p$ denotes the projection of $u$ onto the finite dimensional space, spanned by the 
first $m$ eigenvectors of $A$ ( lower modes ) and $q$ the projection of $u$ onto the infinite dimensional space spanned by the remaining eigenvectors ( higher modes ),

$$
\begin{aligned}
& \frac{d p}{d t}+A p+P B(p+q)=P f \\
& \frac{d q}{d t}+A q+Q B(p+q)=Q f .
\end{aligned}
$$

$P$ and $Q$ are the projectors onto the finite and infinite dimensional spaces. The results of Foias, Manley and Temam ( cf.[6] ) show that a reasonable approximation of $(0.3)$ is given by

$$
A q+Q B(p)=Q f .
$$

This led them to introduce the finite dimensional manifold $M_{0}$ by :

$$
q=\Phi_{0}(p)=A^{-1}(Q f-Q B(p))
$$

Another type of approximate inertial manifolds for two-dimensional turbulent flows containing all stationary solutions has been examined by Titi. ( cf.[12] )

The fact that the two-dimensional MHD-equations have finite dimensional global, universal attractors and a finite number of determining parameters (cf.[1],[8],[9]), inspires us to justify the definition of an approximate inertial manifold similiar to (0.5) for two-dimensional MHD-equations.

The main purpose of this paper is to show that this manifold yields a better approximation than the linear Galerkin approximation.

The plan for our paper is the following.

In section 1 we describe the problem under consideration whereas in section 2 we give a precise functional setting of the problem. Section 3 is related to a few existence and uniqueness results. In section 4 and 5 the main results are presented: norm-estimates for higher modes of the solutions to the MHD-equations and estimates of the distance between the attractor and the approximate inertial manifold. 


\section{The magnetohydrodynamic equations}

The equations, we are interested in, are given in a bounded domain $\Omega \subset \mathbb{R}^{2}$, occupied by the viscous, incompressible and resistive fluid. The unknown functions $u, B$ and $p$ denote the velocity of the fluid, the magnetic field and the pressure of the fluid, respectively.

We shall suppose that the density $\rho$ at time $t=0$ is equal to a prescribed constant $\rho_{0}$. The incompressibility yields that the fluid is homogenouos at time $t \geq 0$. For the sake of simplicity we set $\rho_{0} \equiv 1$.

The nondimensional form of the MHD-equations for viscous, incompressible flows is the following ( $\mathrm{cf} .[2],[8]$ ):

$$
\begin{gathered}
\frac{\partial u}{\partial t}+(u \cdot \nabla) u-\frac{1}{R e} \Delta u+\nabla p+S \nabla\left(\frac{1}{2} B^{2}\right)-S(B \cdot \nabla) B=f \quad \text { in } \Omega \\
\frac{\partial B}{\partial t}+(u \cdot \nabla) B-(B \cdot \nabla) u-\frac{1}{R m} \Delta B=0 \quad \text { in } \Omega
\end{gathered}
$$

$$
\operatorname{div} u=0 \quad \text { in } \Omega, \quad \operatorname{div} B=0 \quad \text { in } \Omega
$$

$p, u, B$ are nondimensional quantities, $f$ represents a nondimensional volume density force. The nondimensional numbers are:

- the Reynolds number $R e$,

- the magnetic Reynolds number $R m$.

- the number $S=M^{2} / R e \cdot R m$, where M is the Hartman number.

We complete (1.1) - (1.3) by initial and boundary conditions upon $u$ and $B$. Let $\partial \Omega$ denote the boundary of $\Omega$ and $\eta$ the unit outward normal along $\partial \Omega$.

$$
\begin{aligned}
& u(x, 0)=u_{0}(x) \quad, \quad B(x, 0)=B_{0}(x) \quad x \in \Omega \\
& u=0 \quad, \quad B \cdot \eta=0 \quad, \quad \operatorname{curl} B=0 \quad \text { on } \partial \Omega
\end{aligned}
$$


Remark:

Since we consider the space dimension $\mathrm{N}=2$, we define the operator curl for every vector function $\varphi=\left(\varphi_{1}, \varphi_{2}\right)$ as follows:

$$
\operatorname{curl} \varphi=\frac{\partial \varphi_{2}}{\partial x_{1}}-\frac{\partial \varphi_{1}}{\partial x_{2}}
$$

The two dimensional case means that the region is a cylinder $\Omega \times \mathbb{R}$, $\Omega \subset \mathbb{R}^{2}$, and all quantities are independent of $x_{3}, u$ and $B$ beeing parallel to the $x_{1}, x_{2}$-plane.

\section{Notations and precise functional setting of the problem}

Let $\Omega \subset \mathbb{R}^{2}$ be a bounded domain with Lipschitz-boundary $\partial \Omega \in C^{0,1}$.By $L^{p}(\Omega), L^{p}\left(\Omega, \mathbb{R}^{2}\right), 1 \leq p \leq \infty ; W^{k, p}(\Omega), W^{k, p}\left(\Omega, \mathbb{R}^{2}\right), 1 \leq p \leq \infty$, $k=1,2, \ldots$; we denote the usual spaces of real valued or $\mathbb{R}^{2}$-valued functions defined on $\Omega$.

$W_{0}^{k, p}(\Omega)$ and $W_{0}^{k, p}\left(\Omega, \mathbb{R}^{2}\right)$ are subspaces of $W^{k, p}(\Omega)$ and $W^{k, p}\left(\Omega, \mathbb{R}^{2}\right)$, respectively, consisting of functions vanishing on $\partial \Omega$.

If there is no danger of misunderstanding we shall write shortly $L^{p}$ for $L^{p}(\Omega)$ as well as for $L^{p}\left(\Omega, \mathbb{R}^{2}\right)$ and $W^{k, p}$ for $W^{k, p}(\Omega)$ as well as for $W^{k, p}\left(\Omega, \mathbb{R}^{2}\right)$. If $E$ is any Banach space and $(0, T)$ an intervall of the real axis we denote by $L^{p}(0, T ; E), 1 \leq p \leq \infty$, the usual space of functions defined on $(0, T)$ with values in $E$. As usual let $E^{\star}$ be the dual space of the Banach space $E$.

Let

$$
(\varphi, \psi):=\int_{\Omega} \varphi(x) \cdot \psi(x) d x
$$

denote the usual scalar product in $L^{2}(\Omega)$.

Next we introduce the spaces, used in the theory of MHD-equations ( cf.[6],[9] ). They are :

$\mathcal{V}_{1}=\left\{\varphi \in C_{c}^{\infty}\left(\Omega, \mathbb{R}^{2}\right) ; \operatorname{div} \varphi=0\right\}$,

$V_{1}=\left\{\varphi \in W_{0}^{1,2}\left(\Omega, \mathbb{R}^{2}\right) ; \operatorname{div} \varphi=0\right\}$ ( the closure of $\mathcal{V}_{1}$ in $\left.W^{1,2}\right)$, 
$\mathcal{V}_{2}=\left\{\Phi \in C^{\infty}\left(\bar{\Omega}, \mathbb{R}^{2}\right) ; \operatorname{div} \Phi=0 ; \Phi \cdot \eta=0 \quad\right.$ on $\left.\partial \Omega\right\}$,

$V_{2}=\left\{\Phi \in W^{1,2}\left(\Omega, \mathbb{R}^{2}\right) ; \operatorname{div} \Phi=0 ; \Phi \cdot \eta=0 \quad\right.$ on $\left.\partial \Omega\right\}$, ( the closure of $\mathcal{V}_{2}$ in $W^{1,2}$ )

$H:=$ the closure of $\mathcal{V}_{1}$ in $L^{2}=$ the closure of $\mathcal{V}_{2}$ in $L^{2}$.

These spaces are equiped with the following scalar products. We define for all $\varphi, \psi \in V_{1}$ :

$$
((\varphi, \psi))_{1}:=\sum_{i=1}^{2}\left(\frac{\partial \varphi}{\partial x_{i}} ; \frac{\partial \psi}{\partial x_{i}}\right),
$$

which is a scalar product in $V_{1}$ and thanks to Friedrich's inequality in $W^{1,2}$. Further we define (cf.[8] ) for all $\Phi, \Psi \in V_{2}$

$$
((\Phi, \Psi))_{2}:=(\operatorname{curl} \Phi, \operatorname{curl} \Psi)
$$

which is a scalar product in $V_{2}$. The norms $\|\cdot\|_{V_{i}}:=\sqrt{((\cdot, \cdot))_{i}}$ in $V_{i}(i=1,2)$ are equivalent to the norm of $W^{1,2}$. So we have :

$$
\begin{array}{ll}
\|\varphi\|_{W^{1,2}} \leq \alpha_{1} \cdot\|\varphi\|_{V_{1}},\|\varphi\|_{V_{1}} \leq \alpha_{2} \cdot\|\varphi\|_{W^{1,2}} & \forall \varphi \in V_{1}, \\
\|\varphi\|_{W^{1,2}} \leq \beta_{1} \cdot\|\varphi\|_{V_{2}},\|\varphi\|_{V_{2}} \leq \beta_{2} \cdot\|\varphi\|_{W^{1,2}} & \forall \varphi \in V_{2}, \\
\alpha_{1}=\alpha_{1}(\Omega), \alpha_{2}=\alpha_{2}(\Omega), \beta_{1}=\beta_{1}(\Omega), \beta_{2}=\beta_{2}(\Omega)=\text { const }>0 .
\end{array}
$$

Finally we mention that the injections

$$
V_{1} \subset H \subset V_{1}^{\star} \quad, \quad V_{2} \subset H \subset V_{2}^{\star}
$$

are compact and continuous and each space is dense in the following.

Let us introduce the operators related to the MHD-equations.

We define two linear continuous operators $\mathcal{A}_{1} \in \mathcal{L}\left(V_{1}, V_{1}^{\star}\right)$ and $\mathcal{A}_{2} \in \mathcal{L}\left(V_{2}, V_{2}^{\star}\right)$ by setting

$$
\begin{aligned}
& \left\langle\mathcal{A}_{1} \varphi, \psi>:=((\varphi, \psi))_{1} \quad \text { for all } \varphi, \psi \in V_{1}\right. \\
& <\mathcal{A}_{2} \Phi, \Psi>:=((\Phi, \Psi))_{2} \quad \text { for all } \Phi, \Psi \in V_{2}
\end{aligned}
$$


We can also consider $\mathcal{A}_{1}$ and $\mathcal{A}_{2}$ as unbounded operators on $H$ whose domains are :

$$
\begin{aligned}
& D\left(\mathcal{A}_{1}\right):=\left\{\varphi \in V_{1}, \mathcal{A}_{1} \varphi \in H\right\}, \\
& D\left(\mathcal{A}_{2}\right):=\left\{\Phi \in V_{2}, \mathcal{A}_{2} \Phi \in H\right\} .
\end{aligned}
$$

In order to characterize $D\left(\mathcal{A}_{1}\right)$ and $D\left(\mathcal{A}_{2}\right)$ we state the following well-known results :

\section{Lemma 2.1:}

Let $g \in V_{1}^{\star}$ be given. Then $\varphi \in V_{1}$ is a solution of $\mathcal{A}_{1} \varphi=g$ if and only if there exists a function $p \in L^{2}(\Omega)$ such that:

$$
-\Delta \varphi+\operatorname{grad} p=g \quad, \quad \operatorname{div} \varphi=0 .
$$

Furthermore if $g \in H$ then there hold $\varphi \in V_{1} \cap W^{2,2}, p \in W^{1,2}$ and there exists a constant $c_{1}=c_{1}(\Omega)$ such that:

$$
\|\varphi\|_{W^{2,2}}+\|p\|_{W^{1,2}} \leq c_{1}\|g\|_{L^{2}} .
$$

Thus we have $D\left(\mathcal{A}_{1}\right)=W^{2,2} \cap V_{1}$ ( cf. [8] ).

\section{Lemma 2.2:}

Let $g \in H$ be given. Then $\Phi \in V_{2}$ is a solution of $\mathcal{A}_{2} \Phi=g$ if and only if $\Phi$ satisfies:

$$
\begin{array}{cc}
-\Delta \Phi=g & \text { in } \Omega, \\
\operatorname{div} \Phi=0 & \text { in } \Omega, \\
\Phi \cdot \eta=0 & \text { on } \partial \Omega, \\
\operatorname{curl} \Phi=0 & \text { on } \partial \Omega .
\end{array}
$$

Furthermore there exists a constant $c_{2}=c_{2}(\Omega)$ such that :

$$
\|\Phi\|_{W^{2,2}} \leq c_{2}\|g\|_{L^{2}} .
$$


Thus we have $D\left(\mathcal{A}_{2}\right)=W^{2,2} \cap V_{2}$ ( cf.[8] ).

The linear operators $\mathcal{A}_{i}: D\left(\mathcal{A}_{i}\right) \subset H \longrightarrow H(i=1,2)$ are symmetric on the real Hilbertspace $H$ with $\operatorname{cl}\left(D\left(\mathcal{A}_{i}\right)\right)=H$ and $\mathcal{A}_{i}$ are strongly monotone, i.e:

$$
\left(\mathcal{A}_{i} \varphi, \varphi\right) \geq c\|\varphi\|^{2} \quad \forall \varphi \in D\left(\mathcal{A}_{i}\right) \text { and fixed } c>0 .
$$

Since the operators $\mathcal{A}_{i}^{-1}: H \longrightarrow H$ are linear, compact and self-adjoint, there exist complete families of eigenvectors $v_{j}$ of $\mathcal{A}_{1}$ and $w_{j}$ of $\mathcal{A}_{2}$, which are orthonormal in $H$ ( cf. [13] ):

$$
\begin{array}{cc}
\mathcal{A}_{1} v_{j}=\lambda_{j} v_{j} & j=1,2, \ldots \\
0<\lambda_{1} \leq \lambda_{2} \leq \ldots & \lambda_{j} \rightarrow \infty \quad \text { as } \quad j \rightarrow \infty, \\
\mathcal{A}_{2} w_{j}=\mu_{j} w_{j} & j=1,2, \ldots \\
0<\mu_{1} \leq \mu_{2} \leq \ldots & \mu_{j} \rightarrow \infty \quad \text { as } \quad j \rightarrow \infty .
\end{array}
$$

Let $P_{m}^{1}$ and $P_{m}^{2}$ denote the projector in $H$ onto the subspace of $H$ spanned by the first $m$ eigenvectors $v_{1}, \ldots, v_{m}$ of $\mathcal{A}_{1}$ and $w_{1}, \ldots, w_{m}$ of $\mathcal{A}_{2}$, respectively. We write $Q_{m}^{1}=I-P_{m}^{1}$ and $Q_{m}^{2}=I-P_{m}^{2}$.

There hold the following well-known estimates:

$$
\begin{aligned}
& \|\varphi\|_{L^{2}} \leq \lambda_{1}^{-1 / 2}\|\varphi\|_{V_{1}} \quad, \quad\|\Phi\|_{L^{2}} \leq \mu_{1}^{-1 / 2}\|\Phi\|_{V_{2}} \\
& \forall \varphi \in V_{1}, \Phi \in V_{2} ; \\
& \|\varphi\|_{V_{1}} \leq \lambda_{1}^{-1 / 2}\left\|\mathcal{A}_{1} \varphi\right\|_{L^{2}} \quad, \quad\|\Phi\|_{V_{2}} \leq \mu_{1}^{-1 / 2}\left\|\mathcal{A}_{2} \Phi\right\|_{L^{2}} \\
& \forall \varphi \in D\left(\mathcal{A}_{1}\right), \Phi \in D\left(\mathcal{A}_{2}\right) ; \\
& \|\varphi\|_{L^{2}} \leq \lambda_{m+1}^{-1 / 2}\|\varphi\|_{V_{1}} \quad, \quad\|\Phi\|_{L^{2}} \leq \mu_{m+1}^{-1 / 2}\|\Phi\|_{V_{2}} \\
& \forall \varphi \in Q_{m}^{1} H, \Phi \in Q_{m}^{2} H \text {; }
\end{aligned}
$$




$$
\begin{gathered}
\|\varphi\|_{V_{1}} \leq \lambda_{m+1}^{-1 / 2}\left\|\mathcal{A}_{1} \varphi\right\|_{L^{2}}, \quad\|\Phi\|_{V_{2}} \leq \mu_{m+1}^{-1 / 2}\left\|\mathcal{A}_{2} \Phi\right\|_{L^{2}} \\
\forall \varphi \in Q_{m}^{1} H, \Phi \in Q_{m}^{2} H
\end{gathered}
$$

We define now a trilinear continuous mapping $b$ on $L^{4}\left(\Omega, \mathbb{R}^{2}\right) \times W^{1,2}\left(\Omega, \mathbb{R}^{2}\right) \times L^{4}\left(\Omega, \mathbb{R}^{2}\right)$ by setting:

$$
b(\varphi, \psi, \xi):=\sum_{i, j=1}^{2} \int_{\Omega} \varphi_{i} \cdot \frac{\partial \psi_{j}}{\partial x_{i}} \cdot \xi_{j} d x .
$$

Due to Hölders inequality this integral makes sense. We have ( cf.[6],[8] ):

$$
\begin{gathered}
b(\varphi, \psi, \psi)=0 \quad \text { for all } \varphi \in V_{2} \quad \psi \in W^{1,2} \\
b(\varphi, \psi, \xi)=-b(\varphi, \xi, \psi) \quad \text { for all } \varphi \in V_{2} \quad \psi, \xi \in W^{1,2}
\end{gathered}
$$

Thanks to (2.1) we can define a continuous bilinear operator $\tilde{\mathcal{B}}$ from $V_{i} \times V_{i}$ into $V_{i}^{\star}(\mathrm{i}=1,2)$ by

$$
<\tilde{\mathcal{B}}(\varphi, \psi), \xi>:=b(\varphi, \psi, \xi),
$$

which satisfies :

$$
\begin{aligned}
&|<\tilde{\mathcal{B}}(\varphi, \psi), \xi>| \leq d_{1} \cdot\|\varphi\|_{L^{4}} \cdot\|\psi\|_{V_{i}} \cdot\|\xi\|_{L^{4}} \\
& \forall \quad \varphi \in L^{4}, \quad \psi \in V_{i}, \quad \xi \in L^{4}, \quad d_{1}=d_{1}(\Omega), \\
&|<\tilde{\mathcal{B}}(\varphi, \psi), \xi>| \leq d_{2} \cdot\|\varphi\|_{L^{\infty}} \cdot\|\psi\|_{V_{i}} \cdot\|\xi\|_{L^{2}} \\
& \forall \varphi \in L^{\infty}, \quad \psi \in V_{i}, \quad \xi \in L^{2}, \quad, \quad d_{2}=d_{2}(\Omega) .
\end{aligned}
$$

This is a simple consequence of Hölders inequality. 


\section{Definition:}

Let $R e, R m, S, T$ be positive real numbers and $(0, T)$ an open intervall of the real axis.

Let $f \in L^{2}\left(0, T ; V_{1}^{\star}\right)$ and $u_{0}, B_{0} \in H$ be given. A tupel $(u, B)$ $u \in W^{1,2}\left(0, T ; V_{1}^{\star}\right) \cap L^{2}\left(0, T ; V_{1}\right), B \in W^{1,2}\left(0, T ; V_{2}^{\star}\right) \cap L^{2}\left(0, T ; V_{2}\right)$ is called weak solution of (1.1) - (1.5) if:

$$
\begin{gathered}
\frac{d}{d t}(u(\tau), \varphi)+\frac{1}{R e}\left\langle\mathcal{A}_{1} u(\tau), \varphi>+<\tilde{\mathcal{B}}(u(\tau), u(\tau)), \varphi>-\ldots\right. \\
\ldots-S<\tilde{\mathcal{B}}(B(\tau), B(\tau)), \varphi>(f(\tau), \varphi) \quad \forall \varphi \in V_{1}, \text { f.a.a. } \tau \in(0, T) \\
\frac{d}{d t}(B(\tau), \Phi)+\frac{1}{R m}<\mathcal{A}_{2} B(\tau), \Phi>+\ldots \\
\ldots+<\tilde{\mathcal{B}}(u(\tau), B(\tau)), \Phi>\quad-\quad<\tilde{\mathcal{B}}(B(\tau), u(\tau)), \Phi>=0 \\
\forall \Phi \in V_{2}, \text { f.a.a. } \tau \in(0, T) \\
u(0)=u_{0} \quad, \quad B(0)=B_{0} .
\end{gathered}
$$

( f.a.a. stands for " for allmost all " )

Remark:

Every function in $L^{2}\left(0, T ; V_{i}\right) \cap W^{1,2}\left(0, T ; V_{i}^{\star}\right) i=1,2$ is continuous on the closed intervall $[0, T]$ with values in $H$. Therefore (2.8) is meaningful. 


\section{Existence, uniqueness and regularity results}

In this section we state an existence result of Temam and Sermange which we need in the following. Furthermore some useful estimates are given.

Theorem 3.1: ( Temam, Sermange cf. [8] )

(i) For $f, u_{0}, B_{0}$ given with $f \in L^{2}\left(0, T ; V_{1}^{\star}\right), u_{0}, B_{0} \in H$ there exists a unique weak solution $(u, B)$ of (1.1) - (1.5).

Furthermore $u$ and $B$ are analytic in $t$ with values in $D\left(\mathcal{A}_{1}\right)$ and $D\left(\mathcal{A}_{2}\right)$, respectively $(t>0)$ and the mapping $u_{0}, B_{0} \longmapsto u(t), B(t)$ is continuous from $H \times H$ into $D\left(\mathcal{A}_{1}\right) \times D\left(\mathcal{A}_{2}\right), \forall t \in(0, T)$.

(ii) If $f \in L^{\infty}(0, T ; H)$ is defined by

$$
f(t):=\tilde{f} \quad, \quad \tilde{f} \in H \quad \text { f.a.a. } t \in(0, T),
$$

$u_{0} \in V_{1}, B_{0} \in V_{2}$, then the weak solution $(u, B)$ satisfies:

$$
\|u(t)\|_{V_{1}} \leq M_{1} \quad, \quad\|B(t)\|_{V_{2}} \leq M_{2} \quad \text { f.a.a. } t \in(0, T),
$$

where $M_{1}, M_{2} \in \mathbb{R}, M_{1}, M_{2}=$ const $>0 ; M_{1}, M_{2}$ are independent of $T$.

(iii) Let $u_{0} \in V_{1}, B_{0} \in V_{2}$ be given and let $f$ be defined as in (3.1) for $0<T \leq \infty$. Then the weak solution to (1.1) - (1.5) satisfies, for every $\alpha_{0}>0: u \in L^{\infty}\left(\alpha_{0}, T ; D\left(\mathcal{A}_{1}\right)\right), B \in L^{\infty}\left(\alpha_{0}, T ; D\left(\mathcal{A}_{2}\right)\right)$ and :

$$
\|u(t)\|_{W^{2,2}} \leq M_{3} \quad, \quad\|B(t)\|_{W^{2,2}} \leq M_{4} \quad \forall t \in\left(\alpha_{0}, T\right),
$$

where $M_{3}, M_{4} \in \mathbb{R} ; M_{3}, M_{4}=$ const $>0 ; M_{3}, M_{4}$ are independent of $T$.

Before turning to the main theorem we note some a-priori estimates.

\section{Lemma 3.2:}

(i) The embedding $W^{1,2}\left(\Omega, \mathbb{R}^{2}\right)$ into $L^{p}\left(\Omega, \mathbb{R}^{2}\right)$ is continuous for $p \in \mathbb{R}$, $1 \leq p<\infty$ and

$$
\|\varphi\|_{L^{p}} \leq \gamma_{1} \quad\|\varphi\|_{W^{1,2}} \quad \forall \varphi \in W^{1,2}\left(\Omega, \mathbb{R}^{2}\right),
$$

$\left(\gamma_{1}\right.$ does not depend on $\varphi$ ). 
(ii) The embedding $W^{2,2}\left(\Omega, \mathbb{R}^{2}\right)$ into $L^{\infty}\left(\Omega, \mathbb{R}^{2}\right)$ is continuous and

$$
\|\varphi\|_{L^{\infty}} \leq \gamma_{2} \quad\|\varphi\|_{W^{2,2}} \quad \forall \varphi \in W^{2,2}\left(\Omega, \mathbb{R}^{2}\right),
$$

$\left(\gamma_{2}\right.$ does not depend on $\left.\varphi\right)$.

Lemma 3.3: (Gronwall's Lemma)

Let $y$ be a positive locally integrable function on $(0, \infty)$ such that $d y / d t$ is locally integrable on $(0, \infty)$, and wich satisfy:

$$
\begin{aligned}
& \frac{d y}{d t}(\tau) \leq a \cdot y(\tau)+b \quad \forall \tau \geq 0 \quad ; \quad a, b=\text { const }>0 \\
& \text { then : } \quad y(\tau) \leq y(0) \cdot \exp (a \cdot \tau)+\frac{b}{a} \quad \forall \tau \geq 0 .
\end{aligned}
$$

Lemma 3.4: (Young's inequality )

$$
a \cdot b \leq \frac{\varepsilon}{p} a^{p}+\frac{1}{q \cdot \varepsilon^{q / p}} b^{q}, \forall a, b, \varepsilon>0,1<p, q<\infty, q=\frac{p}{p-1} .
$$

\section{Statement and Proof of the main theorem}

From now on we assume $f$ to be defined as in (3.1), $u_{0} \in V_{1}$ and $B_{0} \in V_{2}$. To state the main theorem we fix an integer $m \in \mathbb{I N}$ and set for every solution $(u, B)$ to $(1.1)-(1.5)$

$$
\begin{array}{clc}
u=p+q & p=P_{m}^{1} u & q=Q_{m}^{1} u, \\
B=P+Q & P=P_{m}^{2} B & Q=Q_{m}^{2} B,
\end{array}
$$

and we show that after a transient period $q$ is small in comparison with $u$ and $Q$ is small in comparison with $B$, supposing that $m$ is sufficiently large. 


\section{Theorem 4.1:}

If $m$ is sufficiently large then for any solution to (1.1) - (1.5) there hold the estimate:

$$
\begin{gathered}
\|q(t)\|_{L^{2}}+\|Q(t)\|_{L^{2}} \leq\left(\|q(0)\|_{L^{2}}+\|Q(0)\|_{L^{2}}^{2}\right) \exp \left(-k_{0} t\right)+\ldots \\
\ldots+L_{0}\left(\lambda_{m+1}, \mu_{m+1}\right) \quad \forall t \geq 0
\end{gathered}
$$

where $k_{0}=\mu_{m+1} \cdot \min \left(\frac{1}{R e} \cdot \frac{\beta_{1}}{\alpha_{1} \beta_{2}}, \frac{1}{R m}\right)$ and $L_{0} \rightarrow 0$ as $m \rightarrow \infty$.

Proof:

We consider (2.6) and (2.7) with test functions $\varphi=q(t)$ and $\Phi=Q(t)$ $\forall t \in(0, T)$ and obtain because of the symmetrie of the projectors:

$$
\begin{gathered}
\frac{1}{2} \frac{d}{d t}\|q\|_{L^{2}}^{2}+\frac{1}{R e}\|q\|_{V_{1}}^{2}=\ldots \\
\ldots=-<\tilde{\mathcal{B}}(u, u), q>+S<\tilde{\mathcal{B}}(B, B), q>+<Q_{m}^{1} f, q> \\
\frac{1}{2} \frac{d}{d t}\|Q\|_{L^{2}}^{2}+\frac{1}{R m}\|Q\|_{V_{2}}^{2}=\ldots \\
\ldots=-<\tilde{\mathcal{B}}(u, B), Q>+<\tilde{\mathcal{B}}(B, u), Q>.
\end{gathered}
$$


Next we add (4.1) and (4.2) and by virtue of (2.4) and Lemma 3.2 we estimate the right hand side :

$$
\begin{aligned}
& \frac{1}{2} \frac{d}{d t}\|q\|_{L^{2}}^{2}+\frac{1}{2} \frac{d}{d t}\|Q\|_{L^{2}}^{2}+\frac{1}{R e}\|q\|_{V_{1}}^{2}+\frac{1}{R m}\|Q\|_{V^{2}}^{2} \leq \ldots \\
& \ldots \leq d_{1}\left(\|u\|_{L^{4}} \cdot\|u\|_{W^{1,2}}+S \cdot\|B\|_{L^{4}} \cdot\|B\|_{W^{1,2}}\right) \cdot\|q\|_{L^{4}}+\ldots \\
& \ldots+d_{1}\left(\|u\|_{L^{4}} \cdot\|B\|_{W^{1,2}}+\|B\|_{L^{4}} \cdot\|u\|_{W^{1,2}}\right) \cdot\|Q\|_{L^{4}}+\ldots \\
& \ldots+\left\|Q_{m}^{1} f\right\|_{L^{2}} \cdot\|q\|_{L^{2}} \leq \ldots \\
& \ldots \leq D_{1}\|q\|_{W^{1,2}}+D_{2}\|Q\|_{W^{1,2}}+\left\|Q_{m}^{1} f\right\|_{L^{2}} \cdot\|q\|_{W^{1,2}} \leq \ldots \\
& \ldots \leq D_{1} \cdot \alpha_{1} \cdot\|q\|_{V_{1}}+D_{2} \cdot \beta_{1} \cdot\|Q\|_{V_{2}}+\alpha_{1} \cdot\left\|Q_{m}^{1} f\right\|_{L^{2}} \cdot\|q\|_{V_{1}} \leq \ldots
\end{aligned}
$$

(Young's inequality )

$$
\begin{aligned}
& \ldots \leq \frac{1}{2} \mu_{m+1}^{1 / 2} \cdot R e \cdot D_{1}^{2} \cdot \alpha_{1}^{2}+\frac{1}{2 \cdot R e \cdot \mu_{m+1}^{1 / 2}}\|q\|_{V_{1}}^{2}+\frac{1}{2} \cdot \mu_{m+1}^{1 / 2} \cdot R m \cdot D_{2}^{2} \cdot \beta_{1}^{2}+\ldots \\
& \ldots+\frac{1}{2 \cdot R m \cdot \mu_{m+1}^{1 / 2}}\|Q\|_{V_{2}}^{2}+\frac{1}{2} \cdot \mu_{m+1}^{1 / 2} \cdot R e \cdot \alpha_{1}^{2} \cdot\left\|Q_{m}^{1} f\right\|_{L^{2}}^{2}+\frac{1}{2 \cdot R e \cdot \mu_{m+1}^{1 / 2}}\|q\|_{V_{1}}^{2},
\end{aligned}
$$

where

$$
D_{1}=2 \cdot d_{1} \cdot \gamma_{1}^{2} \cdot\left(M_{1}^{2}+S \cdot M_{1} \cdot M_{2}\right)
$$




$$
D_{2}=4 \cdot d_{1} \cdot S \cdot \gamma_{1}^{2} \cdot M_{1} \cdot M_{2}
$$

From (4.1) and (4.2) we conclude:

$$
\begin{gathered}
\frac{d}{d t}\|q\|_{L^{2}}^{2}+\frac{d}{d t}\|Q\|_{L^{2}}^{2}+\ldots \\
\ldots+\|q\|_{V_{1}}^{2}\left(\frac{2}{R e}-\frac{2}{R e} \mu_{m+1}^{-1 / 2}\right)+\|Q\|_{V_{2}}^{2}\left(\frac{2}{R m}-\frac{1}{R m} \mu_{m+1}^{-1 / 2}\right) \leq \ldots \\
\ldots \leq \mu_{m+1}^{1 / 2}\left(\alpha_{1}^{2} \cdot R e \cdot D_{1}^{2}+\beta_{1}^{2} \cdot R m \cdot D_{2}^{2} \cdot+\alpha_{1}^{2} \cdot R e \cdot\left\|Q_{m}^{1} f\right\|_{L^{2}}^{2}\right) .
\end{gathered}
$$

Hence, assuming that $m$ is sufficiently large the last inequality yields:

$$
\begin{gathered}
\frac{d}{d t}\|q\|_{L^{2}}^{2}+\frac{d}{d t}\|Q\|_{L^{2}}^{2}+\ldots \\
\ldots+\mu_{m+1} \cdot \min \left(\frac{1}{R e} \cdot \frac{\beta_{1}}{\alpha_{1} \cdot \beta_{2}}, \frac{1}{R m}\right)\left(\|q\|_{L^{2}}^{2}+\|Q\|_{L^{2}}^{2}\right) \leq \ldots \\
\ldots \leq \mu_{m+1}^{1 / 2}\left(\alpha_{1}^{2} \cdot R e \cdot D_{1}^{2}+\beta_{1}^{2} \cdot R m \cdot D_{2}^{2}+\alpha_{1}^{2} \cdot R e \cdot\left\|Q_{m}^{1} f\right\|_{L^{2}}^{2}\right) .
\end{gathered}
$$

Applying Gronwall's Lemma we obtain:

$\|q(t)\|_{L^{2}}^{2}+\|Q(t)\|_{L^{2}}^{2} \leq\left(\|q(0)\|_{L^{2}}^{2}+\|Q(0)\|_{L^{2}}^{2}\right) \exp \left(-k_{0} t\right)+\ldots$ 


$$
\begin{gathered}
\ldots+\frac{1}{k_{0}} \cdot \mu_{m+1}^{1 / 2}\left(\alpha_{1}^{2} \cdot R e \cdot D_{1}^{2}+\beta_{1}^{2} \cdot R m \cdot D_{2}^{2}+\alpha_{1}^{2} \cdot R e \cdot\left\|Q_{m}^{1} f\right\|_{L^{2}}^{2}\right) \\
\quad \forall t \geq \alpha_{0}>0, \text { where } k_{0}=\mu_{m+1} \cdot \min \left(\frac{1}{R e} \cdot \frac{\beta_{1}}{\alpha_{1} \cdot \beta_{2}}, \frac{1}{R m}\right) .
\end{gathered}
$$

\section{Theorem 4.2:}

If $m$ is sufficiently large then for any weak solution to (1.1) - (1.5) there hold the estimates:

(i)

$$
\|q(t)\|_{V_{1}}^{2} \leq M_{1}^{2} \cdot \exp \left(-t \cdot \lambda_{m+1}\right)+\frac{1}{k_{1}} \cdot L_{1}\left(\lambda_{m+1}\right)
$$

$\forall t \geq \alpha_{0}>0$, where $L_{1} \longrightarrow 0$ as $m \longrightarrow \infty$,

(ii)

$$
\|Q(t)\|_{V_{2}}^{2} \leq M_{2}^{2} \cdot \exp \left(-t \cdot \mu_{m+1}\right)+\frac{1}{k_{2}} \cdot L_{2}\left(\mu_{m+1}\right)
$$

$\forall t \geq \alpha_{0}>0$, where $L_{2} \longrightarrow 0$ as $m \longrightarrow \infty$.

Proof:

ad (i) : Since $u$ ( and so $q$ ) are analytic in $t$ with values in $D\left(\mathcal{A}_{1}\right)(2.6)$ yields :

$$
\frac{d}{d t} u(t)+\frac{1}{R e} \mathcal{A}_{1} u(t)+\hat{\mathcal{B}}(u(t), u(t))-S \cdot \hat{\mathcal{B}}(B(t), B(t))=f \text { in } L^{2} .
$$

Taking the scalar product of $(4.3)$ with $\mathcal{A}_{1} q(t)$ in $L^{2}$ for all $t \in\left(\alpha_{0}, T\right)$ we 
find:

$$
\begin{gathered}
\frac{1}{2} \frac{d}{d t}\|q\|_{V_{1}}^{2}+\frac{1}{R e}\left\|\mathcal{A}_{1} q\right\|_{L^{2}}^{2}=\ldots \\
\ldots=-\left(\hat{\mathcal{B}}(u, u), \mathcal{A}_{1} q\right)+S \cdot\left(\hat{\mathcal{B}}(B, B), \mathcal{A}_{1} q\right)+\left(Q_{m}^{1} f, \mathcal{A}_{1} q\right) .
\end{gathered}
$$

By virtue of (2.4), (2.5) we obtain :

$$
\begin{gathered}
\frac{1}{2} \frac{d}{d t}\|q\|_{V_{1}}^{2}+\frac{1}{R e}\left\|\mathcal{A}_{1} q\right\|_{L^{2}}^{2} \leq \ldots \\
\ldots \leq d_{1} \cdot\|u\|_{L^{\infty}} \cdot\|u\|_{V_{1}} \cdot\left\|\mathcal{A}_{1} q\right\|_{L^{2}}+\ldots
\end{gathered}
$$

$\ldots+d_{1} \cdot S \cdot\|u\|_{L^{\infty}} \cdot\|B\|_{V_{2}} \cdot\left\|\mathcal{A}_{1} q\right\|_{L^{2}}+\left\|Q_{m}^{1} f\right\|_{L^{2}} \cdot\left\|\mathcal{A}_{1} q\right\|_{L^{2}} \leq \ldots$

( Young's inequality )

$$
\ldots \leq \frac{1}{2} D_{3}^{2} \cdot \lambda_{m+1}^{1 / 2} \cdot R e+\frac{1}{2 \cdot \lambda_{m+1}^{1 / 2} \cdot R e}\left\|\mathcal{A}_{1} q\right\|_{L^{2}}^{2}
$$

where

$$
D_{3}=d_{1} \cdot \gamma_{2}\left(M_{3} \cdot M_{1}+S \cdot M_{3} \cdot M_{2}\right)+\left\|Q_{m}^{1} f\right\|_{L^{2}} .
$$

We conclude now :

$$
\frac{d}{d t}\|q\|_{V_{1}}^{2}+\left\|\mathcal{A}_{1} q\right\|_{L^{2}}^{2} \cdot\left(\frac{2}{R e}-\frac{1}{R e} \cdot \lambda_{m+1}^{-1 / 2}\right) \leq D_{3}^{2} \cdot \lambda_{m+1}^{1 / 2} \cdot R e
$$

and if $m$ is sufficiently large :

$$
\frac{d}{d t}\|q\|_{V_{1}}^{2}+\lambda_{m+1} \cdot\|q\|_{V_{1}}^{2} \cdot \frac{1}{R e} \leq D_{3}^{2} \cdot \lambda_{m+1}^{1 / 2} \cdot R e .
$$


Applying Gronwalls Lemma we obtain :

$$
\begin{gathered}
\|q(t)\|_{V_{1}}^{2} \leq\left\|q\left(\alpha_{0}\right)\right\|_{V_{1}}^{2} \cdot \exp \left(-t \cdot \lambda_{m+1}\right)+\frac{D_{3}^{2} \cdot R e}{\lambda_{m+1}^{1 / 2}} \leq \ldots \\
\ldots \leq M_{1}^{2} \exp \left(-t \cdot \lambda_{m+1}\right)+\frac{D_{3}^{2} \cdot R e}{\lambda_{m+1}^{1 / 2}} \quad \forall t \geq \alpha_{0}>0 .
\end{gathered}
$$

ad (ii) : Since $B$ ( and so $Q$ ) are analytic in $t$ with values in $D\left(\mathcal{A}_{2}\right)$ we infer from $(2.7)$ :

$\frac{d}{d t} B(t)+\frac{1}{R m} \mathcal{A}_{2} B(t)+\hat{B}(u(t), B(t))-\hat{B}(B(t), u(t))=0 \quad$ in $\mathrm{E}^{2}$.

Taking the scalar product of (4.5) with $\mathcal{A}_{2} Q(t)$ for all $t \in\left(\alpha_{0}, T\right)$ we find :

$$
\begin{gathered}
\frac{1}{2} \frac{d}{d t}\|Q\|_{V_{2}}^{2}+\frac{1}{R m}\left\|\mathcal{A}_{2} Q\right\|_{L^{2}}^{2}=\ldots \\
\ldots-\left(\hat{\mathcal{B}}(u, B), \mathcal{A}_{2} Q\right)+\left(\hat{\mathcal{B}}(B, u), \mathcal{A}_{2} Q\right) .
\end{gathered}
$$

By virtue of (2.5) Lemma 3.2 we obtain :

$$
\begin{gathered}
\frac{1}{2} \frac{d}{d t}\|Q\|_{V_{2}}^{2}+\frac{1}{R m}\left\|\mathcal{A}_{2} Q\right\|_{L^{2}}^{2} \leq d_{2} \cdot\|u\|_{L^{\infty}} \cdot\|B\|_{V_{2}} \cdot\left\|\mathcal{A}_{2} Q\right\|_{L^{2}}+\ldots \\
\ldots+d_{2} \cdot\|B\|_{L^{\infty}} \cdot\|u\|_{V_{1}} \cdot\left\|\mathcal{A}_{2} Q\right\|_{L^{2}} \leq \ldots
\end{gathered}
$$

(Young's inequality )

$$
\ldots \leq \frac{1}{2} \mu_{m+1}^{1 / 2} \cdot R m \cdot D_{4}^{2}+\frac{1}{2 \cdot \mu_{m+1}^{1 / 2} \cdot R m}\left\|\mathcal{A}_{2} Q\right\|_{L^{2}}
$$

where $\quad D_{4}=d_{2} \cdot \gamma_{2} \cdot\left(M_{3} \cdot M_{2}+M_{4} \cdot M_{1}\right)$. 
Therefore :

$$
\frac{d}{d t}\|Q\|_{V_{2}}^{2}+\left\|\mathcal{A}_{2} Q\right\|_{L^{2}}^{2} \cdot\left(\frac{2}{R m}-\frac{1}{R m \cdot \mu_{m+1}^{1 / 2}}\right) \leq \mu_{m+1}^{1 / 2} \cdot R e \cdot D_{4}^{2}
$$

and if $\mathrm{m}$ is sufficiently large :

$$
\frac{d}{d t}\|Q\|_{V_{2}}^{2}+\mu_{m+1}\|Q\|_{V_{2}}^{2} \cdot \frac{1}{R m} \leq \mu_{m+1}^{1 / 2} \cdot R e \cdot D_{4}^{2}
$$

Applying Gronwalls Lemma we obtain :

$$
\begin{gathered}
\|Q(t)\|_{V_{2}}^{2} \leq\left\|Q\left(\alpha_{0}\right)\right\|_{V_{2}}^{2} \cdot \exp \left(-t \cdot \mu_{m+1}\right)+\frac{R e \cdot D_{4}^{2}}{\mu_{m+1}^{1 / 2}} \leq \ldots \\
\ldots \leq M_{2}^{2} \cdot \exp \left(-t \cdot \mu_{m+1}\right)+\frac{R e \cdot D_{4}^{2}}{\mu_{m+1}^{1 / 2}} \quad \forall t \geq \alpha_{0}>0 .
\end{gathered}
$$

\section{The approximate inertial manifold}

As mentioned in the introduction we are going to improve the linear Galerkinapproximation from section 4 by definition of a finite dimensional manifold $\mathcal{M}_{0}$.

We consider the projection $u=p+q$ and $B=P+Q$ for any solution to (1.1) - (1.5) and write these equations equivalently as a coupled system of equations for $p, q$ and $P, Q$ :

$$
\begin{gathered}
\frac{d}{d t} p(t)+\frac{1}{R e} \mathcal{A}_{1} p(t)+P_{m}^{1} \tilde{\mathcal{B}}(p(t)+q(t), p(t)+q(t))-\ldots \\
\ldots-S \cdot P_{m}^{1} \tilde{\mathcal{B}}(P(t)+Q(t), P(t)+Q(t))=P_{m}^{1} f \quad \text { in } L^{2} \forall t \in(0, T) \\
\frac{d}{d t} q(t)+\frac{1}{R e} \mathcal{A}_{1} q(t)+Q_{m}^{1} \tilde{\mathcal{B}}(p(t)+q(t), p(t)+q(t))-\ldots
\end{gathered}
$$




$$
\begin{gathered}
\ldots-S \cdot Q_{m}^{1} \tilde{\mathcal{B}}(P(t)+Q(t), P(t)+Q(t))=Q_{m}^{1} f \quad \text { in } L^{2} \forall t \in(0, T) \\
\frac{d}{d t} P(t)+\frac{1}{R m} \mathcal{A}_{2} P(t)+P_{m}^{2} \tilde{\mathcal{B}}(p(t)+q(t), P(t)+Q(t))+\ldots \quad(5.3) \\
\ldots+P_{m}^{2} \tilde{\mathcal{B}}(P(t)+Q(t), p(t)+q(t))=0 \quad \text { in } L^{2} \forall t \in(0, T) \\
\frac{d}{d t} Q(t)+\frac{1}{R m} \mathcal{A}_{2} Q(t)+Q_{m}^{2} \tilde{B}(p(t)+q(t), P(t)+Q(t))+\ldots \quad(5.4) \\
\ldots+Q_{m}^{2} \tilde{\mathcal{B}}(P(t)+Q(t), p(t)+q(t))=0 \quad \text { in } L^{2} \forall t \in(0, T)
\end{gathered}
$$

The results of chapter 4 show that an acceptable approximation to (5.2) and (5.4) is given by :

$$
\frac{1}{R e} \mathcal{A}_{1} q(t)+Q_{m}^{1} \tilde{\mathcal{B}}(p(t), p(t))-S \cdot Q_{m}^{1} \tilde{B}(P(t), P(t))=Q_{m}^{1} f
$$

and

$$
\frac{1}{R m} \mathcal{A}_{2} Q(t)+Q_{m}^{2} \tilde{\mathcal{B}}(p(t), P(t))+Q_{m}^{2} \tilde{\mathcal{B}}(P(t), p(t))=0 .
$$

So we are able to introduce in $H \times H$ the finite dimensional manifold $\mathcal{M}_{0}$. We define a mapping $\Phi_{0}: P_{m}^{1} H \times P_{m}^{2} H \longrightarrow Q_{m}^{1} H \times Q_{m}^{2} H$ by setting :

$$
\begin{gathered}
\left(q_{m}, Q_{m}\right)=\Phi_{0}(p, P):=\ldots \\
\ldots=\mathcal{A}_{1}^{-1}\left(\operatorname{Re}\left[Q_{m}^{1} f+S \cdot Q_{m}^{1} \tilde{\mathcal{B}}(P, P)-Q_{m}^{1} \tilde{\mathcal{B}}(p, p)\right]\right) \\
\mathcal{A}_{2}^{-1}\left(R m\left[-Q_{m}^{2} \tilde{\mathcal{B}}(P, p)-Q_{m}^{2} \tilde{\mathcal{B}}(p, P)\right]\right) .
\end{gathered}
$$


The manifold $\mathcal{M}_{0}$ is defined by :

$$
\mathcal{M}_{0}:=\left\{(p, P)+\Phi_{0}(p, P) ; \quad p \in P_{m}^{1} H, P \in P_{m}^{2} H\right\} .
$$

From (5.2) and (5.4) we infer :

$$
\begin{array}{ll}
\left\|\frac{d}{d t} q(t)\right\|_{L^{2}} \leq M_{5} & \forall t \in[0, T], \\
\left\|\frac{d}{d t} Q(t)\right\|_{L^{2}} \leq M_{6} & \forall t \in[0, T] .
\end{array}
$$

$M_{5}, M_{6}=$ const $>0 ; M_{5}$ and $M_{6}$ are independent of $\mathrm{T}$.

Estimating the distance of $(u(t), B(t))$ to $\mathcal{M}_{o}$ means to estimate the distance of $(q, Q)$ to $\left(q_{m}, Q_{m}\right)$. To end this we subtract $(q, Q)$ from $\left(q_{m}, Q_{m}\right)$, using $(5.2),(5.4)$ and the definition of $\Phi_{0}$ and obtain:

$$
\begin{gathered}
\frac{1}{R e} \cdot \lambda_{m+1} \cdot\left\|q_{m}(t)-q(t)\right\|_{L^{2}} \leq \ldots \\
\frac{1}{R e} \cdot \lambda_{m+1} \cdot\left\|q_{m}(t)-q(t)\right\|_{V_{1}} \leq \frac{1}{R e} \cdot\left\|\mathcal{A}_{1} q_{m}(t)-\mathcal{A}_{1} q(t)\right\|_{L^{2}}=\ldots \\
\ldots=S \cdot\left\|Q_{m}^{1} \tilde{\mathcal{B}}(P(t), P(t))-Q_{m}^{1} \tilde{\mathcal{B}}(B(t), B(t))\right\|_{L^{2}}+\ldots \\
\left\|Q_{m}^{1} \tilde{\mathcal{B}}(p(t), p(t))-Q_{m}^{1} \mathcal{B}(u(t), u(t))\right\|_{L^{2}}+\left\|\frac{d}{d t} q(t)\right\|_{L^{2}} \leq \ldots \\
\ldots \leq S \cdot d_{1} \cdot \gamma_{1} \cdot 2 M_{2}^{2}+d_{1} \cdot \gamma_{1} \cdot 2 M_{1}^{2}+M_{5}=: D_{5} ;
\end{gathered}
$$




$$
\begin{gathered}
\frac{1}{R m} \cdot \mu_{m+1} \cdot\left\|Q_{m}(t)-Q(t)\right\|_{L^{2}} \leq \\
\leq \frac{1}{R m} \cdot \mu_{m+1} \cdot\left\|Q_{m}(t)-Q(t)\right\|_{V_{2}} \leq \frac{1}{R m} \cdot\left\|\mathcal{A}_{2} Q_{m}(t)-\mathcal{A}_{2} Q(t)\right\|_{L^{2}}=\ldots \\
\ldots=\left\|Q_{m}^{2} \tilde{\mathcal{B}}(P(t), p(t))-Q_{m}^{2} \mathcal{B}(B(t), u(t))\right\|_{L^{2}}+\ldots \\
\ldots+\left\|Q_{m}^{2} \mathcal{B}(p(t), P(t))-Q_{m}^{2} \mathcal{B}(u(t), B(t))\right\|_{L^{2}}+\left\|\frac{d}{d t} Q(t)\right\|_{L^{2}} \leq \\
\ldots \leq d_{1} \gamma_{1} 2 M_{2} \cdot M_{1}+d_{1} \cdot \gamma_{1} 2 M_{1} \cdot M_{2}+M_{6}=: D_{6} .
\end{gathered}
$$

So we have :

$$
\left\|q_{m}(t)-q(t)\right\|_{L^{2}} \leq \lambda_{m+1}^{-1} \cdot R e \cdot D_{5}
$$

and

$$
\left\|Q_{m}(t)-Q(t)\right\|_{L^{2}} \leq \mu_{m+1}^{-1} \cdot R m \cdot D_{6}
$$

\section{Summary}

A new method of approximating the solutions of the magnetohydrodynamicequations for a long time by means of approximate inertial manifolds has been proposed.

This approximation scheme has been derived directly from the MHD-equations without any phenomenological considerations.

The last two inequalities of section 5 show that the distance between any solution to the MHD-equations and the approximate inertial manifold is smaller than the distance to the flat space $q=0$ by a factor $\lambda_{m+1}^{-1 / 2}$ for the 
velocity and $\mu_{m+1}^{-1 / 2}$ for the magnetic field.

Our arguments have been yielded an improvement of the distance to the manifold $\mathcal{M}_{0}$ in the $L^{2}$-norm. The estimates in the $W^{1,2}$-norm will be one of our subjects for further investigation.

Acknowledgement. -The author is indebted to N.Seehafer and F.Feudel for a number of useful discussions when preparing this paper.

\section{References}

1. Eden A. \& Libin A., Explicit Dimension Estimates of attractors for the MHD-Equations in three dimensional space, Physica D 40,1989 , pp.338352, North Holland, Amsterdam

2. Cowling T.G., Magnetohydrodynamics, Interscience Tracts on Physics and Astronomy, New York 1957

3. Constantin P. \& Foias C. \& Nicolaenko B. \& Temam R., Integral Manifolds and Inertial Manifolds for Dissipative Partial Differential Equations, Springer-Verlag New York, 1989

4. Constatin P. \& Foias C. \& Temam R., On the large time Galerkin approximation of the Navier-Stokes-Equations, SIAM J.Numerical Analysis 21 , 1984, pp.615-634

5. Foias C. \& Temam R., Some analytic and geometric properties of the solutions of the Navier-Stokes-Equations, J.Math.Pures Appl. 58 , 1979. pp.339-368

6. Foias C. \& Manley O. \& Temam R.,

Modelling of the interaction of small and large eddies in two dimensional turbulent flows, Mathematical Modelling and Numerical Analysis, 22 , 1988, pp.93-114

7. Foias C. \& Manley O. \& Temam R. \& Treve Y.M., Asymtotic Analysis of the Navier-Stokes-Equations, Physica 9D , 1983, pp.157-188 North Holland Publishing Company

8. Sermange M. \& Temam R., Some Mathematical Questions Related to the MHD-Equations, Comm. on Pure and Applied Mathematics, Vol.XXXVI, 1983, pp.635-664

9. Temam R., Infinite Dimensional Dynamical Systems in Mechanics and Physics, Springer-Verlag New York, 1988

10. Temam R., Approximation of attractors, large eddy simulation and 
multiscal methods, Proc.R.Soc.Lond.A , 434 , 1991, pp.23-39

11. Shubov V.I., Long Time Behavior of Infinite Dimensional Dissipative Dynamical Systems, Journal of Mathematical Systems, Estimation and Control , Vol.2 , No.4, 1992, pp.381-427

12. Titi S., On Approximate Inertial Manifolds to the Navier Stokes Equations, Journal of mathematical Analysis and Applications , 149 , 1990. pp. $540-557$

13. Zeidler E., Nonlinear Functional Analysis and its Applications II/A, Springer-Verlag New York, 1990

14. Marion M. \& Temam R., Nonlinear Galerkin Methods, SIAM J.Num.Anal., Vol.26, No.5, pp.1139-1157, 1989

15. Foias C. \& Sell G.R. \& Temam R., Inertial Manifolds for Nonlinear Evolutionary Equations, Journal of Differential Equations 73, pp.309-353, 1989 